\title{
Efectos de la aplicación de mulch para el control de la erosión post-incendio sobre la recuperación de la vegetación en áreas de matorral
}

\author{
Fernández C..$^{1 *}$, Vega J. A. ${ }^{1}$ \\ ${ }^{1}$ Centro de Investigación Forestal de Lourizán, Xunta de Galicia, Apdo. 127, 36080, Pontevedra \\ * e-mail: cffilgueira@gmail.com
}

\section{Resumen}

La aplicación de mulching de paja u otros materiales después de incendio se ha mostrado como un tratamiento eficaz para reducir las pérdidas de suelo por erosión. Sin embargo, esta cubierta puede afectar a la regeneración natural de la vegetación. Además, el uso de paja de origen agrícola podría aumentar el riesgo de invasión de plantas alóctonas. En este trabajo se resumen los resultados de la investigación llevada a cabo en el Centro de Investigación Forestal de Lourizán desde mediados de la década de 2000 en la que se ha ensayado el efecto de la aplicación de mulch de paja agrícola, astillas y corteza desfibrada de eucalipto en áreas incendiadas sobre la cobertura vegetal y la composición de especies en áreas de matorral de Galicia. Los resultados indican que en áreas de influencia oceánica donde la precipitación supera los $1500 \mathrm{~mm}$, la adición de mulch no tiene efecto sobre la regeneración natural de la vegetación. Sin embargo, en el interior de Galicia donde las condiciones microclimáticas son más adversas, el mulching favorece tanto la emergencia de plántulas de semilla como el rebrote de la vegetación. En ningún caso se observó un cambio en la composición de especies ni un aumento significativo de la presencia de especies exóticas, si bien debe tenerse en cuenta el origen del mulch para reducir posibles efectos indeseables en la vegetación autóctona.

Palabras clave: incendio, mulching, plantas alóctonas, regeneración natural. 


\section{Introducción}

El aumento de las pérdidas de suelo por erosión es una de las consecuencias más dramáticas de los incendios forestales. El fuego puede destruir total o parcialmente la cubierta orgánica muerta del suelo y la vegetación, modificando las condiciones de infiltración, exponiendo el suelo al impacto de la lluvia o creando condiciones de hidrofobicidad (De Bano et al., 1998; Neary et al., 2005). La movilización de sedimentos desde las laderas puede provocar un impacto significativo en los ecosistemas, particularmente sobre los ríos, lagos y zonas ripícolas lo que puede alterar la calidad del agua además de inducir cambios en la características geomorfológicas e hidrológicas de esos sistemas y afectar su fertilidad futura (Shakesby and Doerr, 2006).

La aplicación urgente de tratamientos de estabilización del suelo en áreas que han sufrido con un alto nivel de severidad del fuego en el suelo es necesaria para minimizar las pérdidas de suelo post-incendio (Napper, 2006; Vega et al., 2013).

El tratamiento a base de cubrir el suelo directamente con restos vegetales (mulching) el impacto directo de la lluvia, reducir el sellado del suelo, favorecer la infiltración y disminuir la escorrentía (Smets et al., 2008). El material más utilizado es la paja de origen agrícola que ha mostrado su eficacia para limitar la erosión post-incendio cuando es aplicada a una dosis igual o superior a $2 \mathrm{Mg} /$ ha (Bautista et al., 1996; Fernández et al., 2011; Fernández y Vega, 2014; Vega et al., 2014; Vega et al., 2015). En los últimos años mulches de distintos tipos de residuos forestales han empezado a utilizarse como alternativas a la paja. Esos materiales son más caros de producir y aplicar pero son menos susceptibles de ser movilizados por el viento y también son más duraderos que el mulch de paja, protegiendo el suelo durante más tiempo (Foltz, 2012).

La información disponible sobre cómo la aplicación de mulch afecta a la recuperación de la vegetación, tanto en términos de cobertura como de composición de especies, es todavía escasa e inconcluyente. Se ha observado tanto inhibición del crecimiento vegetal (Beyers, 2004; Kruse et al., 2004; Dodson and Peterson, 2010), como su estimulación, al mejorar las condiciones microclimáticas (Bautista et al., 2009; Peterson et al., 2009). No existe un nivel de información similar para los mulches de residuos forestales aunque los resultados disponibles hasta ahora parecen indicar un efecto neutro sobre la vegetación (Foltz, 2012; Robichaud et al., 2013).

El uso de paja agrícola como material para el mulch podría alterar la composición de especies vegetales al facilitar la introducción de especies alóctonas (Kruse et al., 2004). Esto parece menos probable cuando se usen mulches de residuos forestales (Foltz, 2012) y en áreas donde la vegetación está muy adaptada a fuegos repetidos, la precipitación es abundante y/o se regenera por rebrote cubriendo rápidamente el suelo. Sin embargo, en zonas donde la vegetación se regenera por semilla, la introducción de semillas de especies alóctonas puede suponer un problema (Hunter et al., 2006).

En el presente trabajo se resumen algunos de los resultados de la investigación llevada a cabo en el Centro de Investigación de Lourizán sobre el efecto de la aplicación de diferentes tipos de mulch en la recuperación de la cubierta vegetal en dife- 
rentes áreas de matorral afectadas por incendio y donde esos tratamientos han sido aplicados. Los principales objetivos fueron:

a) determinar el efecto de la aplicación de mulch sobre la recuperación de la cobertura vegetal.

b) analizar si la aplicación de mulch altera el número de especies o favorece la aparición de especies alóctonas en esas comunidades vegetales.

\section{Material y métodos}

El estudio se llevó cabo en cuatro áreas de matorral afectadas por incendio en las que se había aplicado mulching para el control de la erosión post-incendio (Tabla 1). En cada sitio experimental se instaló un número variable de parcelas (de entre 80 y $500 \mathrm{~m}^{2}$ ) siguiendo un diseño de bloques al azar.

Tabla 1. . Características generales de los sitios experimentales

\begin{tabular}{|c|c|c|}
\hline Localización & $\begin{array}{l}\text { Principales especies comunidad } \\
\text { vegetal previa al incendio }\end{array}$ & Tratamientos $/ \mathrm{n}^{\circ}$ réplicas \\
\hline $\begin{array}{l}\text { Soutelo } \\
\text { de Montes } \\
\text { (Pontevedra) }\end{array}$ & $\begin{array}{l}\text { Ulex europaeus L. } \\
\text { Erica cinerea L. } \\
\text { Daboecia cantabrica (Huds.) K. Koch }\end{array}$ & $\begin{array}{l}\text { Mulch paja }\left(250 \mathrm{~g} \mathrm{~m}^{-2}\right) / 4 \\
\text { Mulch astillas }\left(400 \mathrm{~g} \mathrm{~m}^{-2}\right) / 4 \\
\text { Control/4 }\end{array}$ \\
\hline $\begin{array}{l}\text { O Irixo } \\
\text { (Ourense) }\end{array}$ & $\begin{array}{l}\text { Pterospartum tridentatum (L.) Willk. } \\
\text { Ulex gallii Planch. } \\
\text { Erica umbellata Loefl. (L.) }\end{array}$ & $\begin{array}{l}\text { Mulch paja }\left(250 \mathrm{~g} \mathrm{~m}^{-2}\right)+\text { siembra } \\
\text { gramíneas dosis baja }\left(3 \mathrm{~g} \mathrm{~m}^{-2}\right) / 5 \\
\text { Control/5 }\end{array}$ \\
\hline $\begin{array}{l}\text { Serra Fial } \\
\text { das Corzas } \\
\text { (Ourense) }\end{array}$ & $\begin{array}{l}\text { Erica australis L. } \\
\text { Pterospartum tridentatum (L.) Willk. }\end{array}$ & $\begin{array}{l}\text { Mulch paja }\left(200 \mathrm{~g} \mathrm{~m}^{-2}\right) / 4 \\
\text { Mulch corteza desfibrada eucalipto } \\
\left(350 \mathrm{~g} \mathrm{~m}^{-2}\right) / 4 \\
\text { Control/4 }\end{array}$ \\
\hline $\begin{array}{l}\text { O Saviñao } \\
\text { (Lugo) }\end{array}$ & $\begin{array}{l}\text { Cytisus striatus (Hill) Rothm. } \\
\text { Pterospartum tridentatum (L.) Willk. } \\
\text { Ulex gallii Planch. }\end{array}$ & $\begin{array}{l}\text { Mulch paja }\left(200 \mathrm{~g} \mathrm{~m}^{-2}\right) / 4 \\
\text { Control/4 }\end{array}$ \\
\hline
\end{tabular}

En cada parcela de cada sitio experimental se situaron al menos diez cuadros de $1 \mathrm{~m}$ de lado para el seguimiento de la cobertura vegetal por especie, registrándose todas las especies presentes. Las mediciones se realizaron durante los dos años siguientes a la ejecución de los tratamientos, con la excepción del dispositivo de $\mathrm{O} \mathrm{Sa}$ viñao en donde el seguimiento de la recuperación de la cubierta vegetal se realizó sólo durante el primer año post-fuego. En este sitio experimental se extrajeron monolitos de suelo que se instalaron en invernadero en condiciones óptimas para la emergen- 
cia de las plántulas y poder comparar el efecto del mulch de paja en las condiciones de campo con el efecto del mulch sin limitaciones de temperatura y humedad.

El efecto del tratamiento sobre la cobertura de la vegetación y riqueza de especies fue testado usando un análisis de varianza en cada sitio experimental al final del período de estudio. En Saviñao, además se comparó la emergencia de plántulas entre mulch y control en invernadero y campo. El cumplimiento de los supuestos de normalidad y homocedasticidad de los residuos implicó la transformación de los datos usando logaritmos. Cuando el efecto tratamiento resultó significativo $(\mathrm{P}<0,05)$ se usó el test de Student-Newman-Keuls para la comparación de medias. El paquete estadístico R (Core Team Development, 2014) fue empleado para llevar a cabo los análisis.

\section{Resultados}

La aplicación del mulch de paja supuso una cobertura inicial del suelo del $80 \%$, como promedio. Como contraste, los mulches de astilla o corteza apenas cubrieron el $50 \%$ del suelo inmediatamente después de su aplicación, a pesar de que las cantidades usadas fueron bastante mayores. La recuperación de la cubierta vegetal fue rápida, superando el $70 \%$ dos años después del incendio en todos los sitios. Ni el mulch de paja ni el de astilla tuvieron una influencia significativa en la recuperación de la cobertura del tojal de Soutelo (Figura 1 a). Sin embargo, en O Irixo, Fial das Corzas y O Saviñao (Figuras $1 b c d$ ) la aplicación de mulch supuso un incremento significativo $(\mathrm{p}<0,05)$ de la cobertura vegetal en comparación con el control no tratado. La cobertura vegetal en el tratamiento de corteza desfibrada de eucalipto no difirió de manera significativa del mulch de paja en Fial das Corzas.

En el experimento de emergencia de O Saviñao, no se observaron diferencias en la densidad de plántulas emergidas por $\mathrm{m}^{-2}$ entre el tratamiento de mulch y control en la población mantenida en invernadero bajo condiciones óptimas de temperatura y humedad $\left(731,5\right.$ plántulas $\mathrm{m}^{-2}$; error estándar $=76,6 v \mathrm{~s}$. 633,7 plántulas $\mathrm{m}^{-2}$; error estándar $=69,3)$ durante el primer año post-incendio. Por el contrario, en el muestreo de campo se encontró que la emergencia de plántulas era superior de forma significativa en el tratamiento de mulch $\left(167,4\right.$ plántulas $\mathrm{m}^{-2}$; error estándar $\left.=12,2\right)$ en comparación con el control no tratado $\left(114,1\right.$ plántulas $\mathrm{m}^{-2}$; error estándar $\left.=16,3\right)$.

En cuanto a la riqueza de especies (Figura 2), no se detectaron diferencias significativas entre tratamientos en ninguno de los sitios experimentales estudiados. Únicamente se observó presencia de Hordeum vulgare y Avena sativa en las parcelas de mulch del dispositivo de O Saviñao.

\section{Discusión}

El efecto neutro o positivo de la aplicación de mulch de paja sobre la recuperación de la cobertura vegetal confirma previos resultados obtenidos en el Oeste de los Estados Unidos, usando una dosis de paja similar, que proporciona una cobertura 

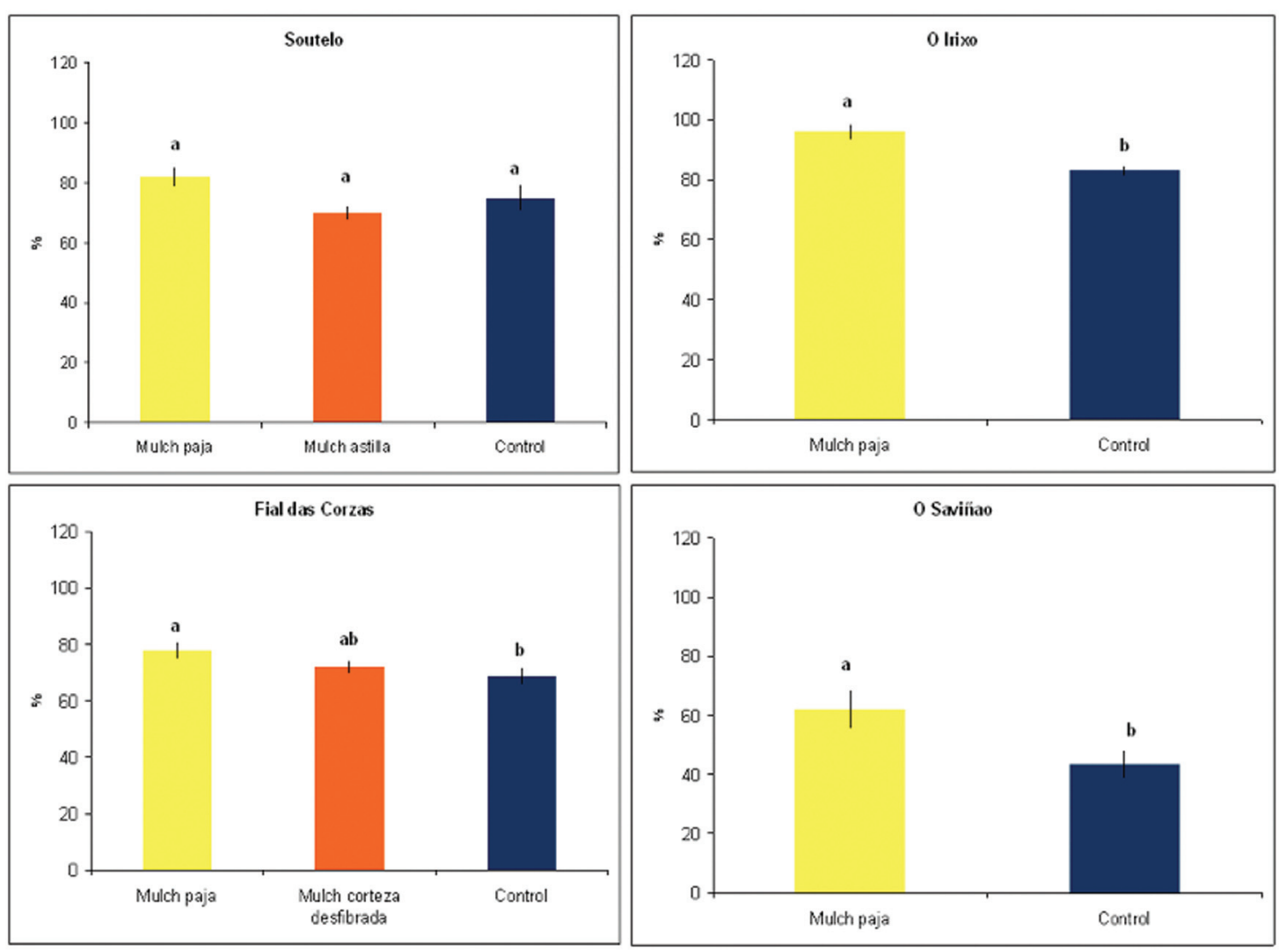

Figura 1. Porcentaje promedio de cobertura vegetal al final del período de estudio. Barras verticales, error estándar. Letras iguales, medias de los valores correspondientes a cada tratamiento no difieren entre sí significativamente $(\mathrm{p}>0,05)$.

media del suelo quemado del 80\% (Peterson et al., 2009). Este resultado puede deberse a unas mejores condiciones de humedad edáfica en el suelo protegido por el mulch, favoreciendo incluso la regeneración por rebrote, en comparación con el suelo desnudo de las áreas sin tratar (Fernández et al., 2013). El estudio comparativo de emergencia entre el campo e invernadero también confirma que el mulching proporciona mejores condiciones para el establecimiento de la cubierta vegetal, en lugares con bajas temperaturas invernales y estrés hídrico estival. Estos resultados son consistentes con el incremento de humedad del suelo y un efecto amortiguador de la temperatura en áreas tratadas con mulch de astillas observado por Santana et al. (2014), aunque estos autores no detectaron ningún efecto sobre la emergencia de plántulas.

Los mulches de astillas o de corteza desfibrada no tuvieron efecto alguno en la recuperación de la cubierta vegetal, probablemente debido a la baja cobertura que proporcionaron después de su aplicación. En ambos casos, aunque la cantidad aplicada fue muy superior a la de la paja, apenas cubrieron un 50\% del suelo quemado. Foltz (2012) y Robichaud et al. (2013) tampoco encontraron que la adición de astillas afectase a la cobertura vegetal. 

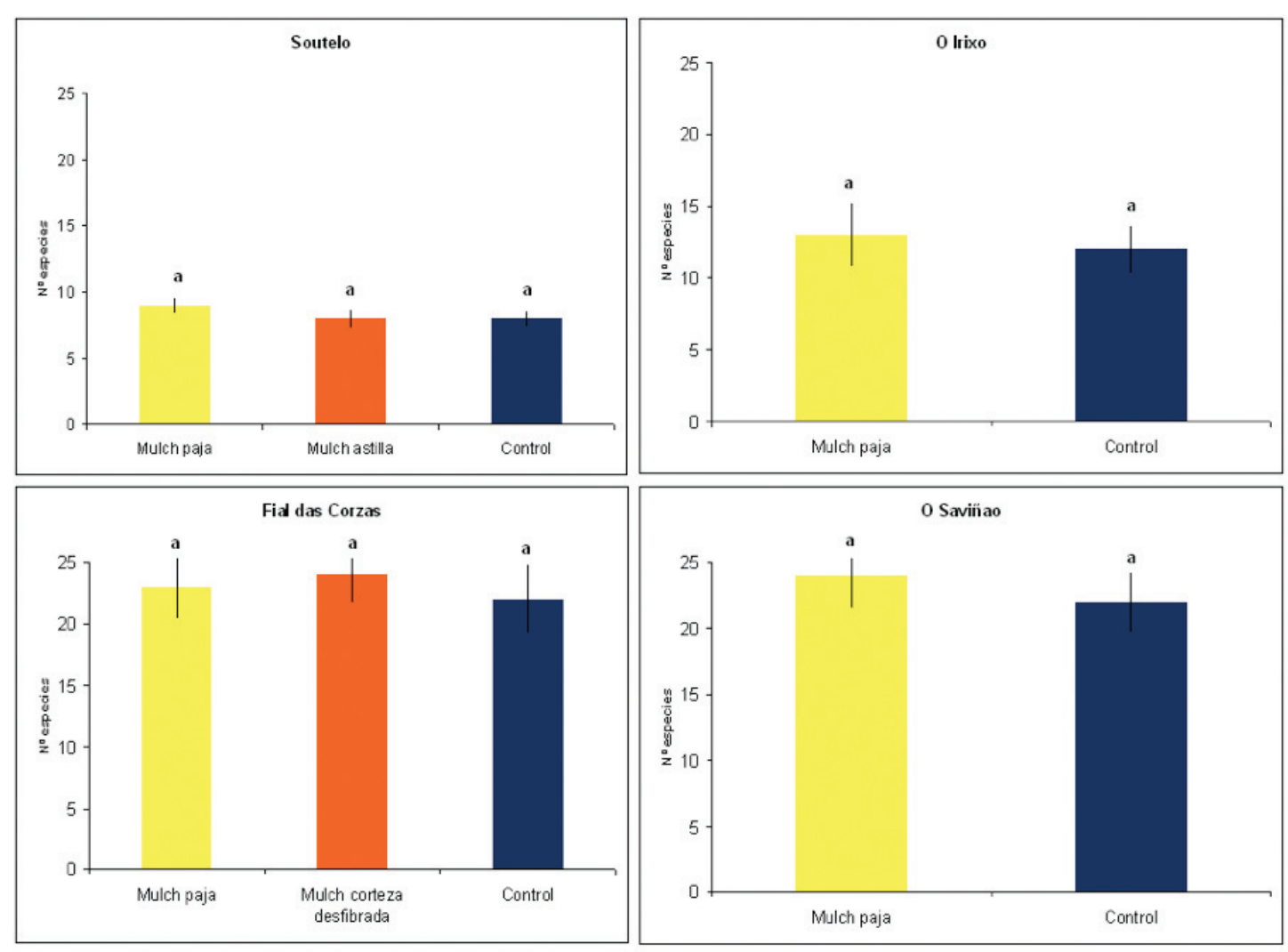

Figura 2. Número de especies promedio en cada tratamiento. Barras verticales, error estándar. Letras iguales, medias de los valores correspondientes a cada tratamiento no difieren entre sí significativamente ( $\mathrm{p}>$ $0,05)$.

No se detectó que la aplicación de mulch influyera en la composición de especies en los sitios estudiados, lo que contrasta con los resultados obtenidos previamente (Kruse et al., 2004; Dodson and Peterson, 2010; Dodson et al., 2010). En el presente estudio solo se observó la presencia de dos especies alóctonas en uno de los sitios experimentales, aunque con bajo riesgo de resultar invasoras (Sanz et al., 2004).

En todos los casos la recuperación de la cubierta vegetal fue muy rápida, debido a que la mayor parte de las especies presentes están adaptadas al fuego (Reyes and Casal, 2008). Dos años después de incendio la cobertura vegetal había alcanzado al menos un $60 \%$, el umbral mínimo para considerar que el suelo está protegido frente a la erosión (Johansen et al., 2001).

\section{Conclusiones}

La adición de mulch de paja tuvo un efecto mayoritariamente positivo sobre la recuperación de la cubierta vegetal. La aplicación de mulches de materiales forestales tuvo un impacto neutro sobre la regeneración de la vegetación preexistente. 
El uso de mulch no favoreció el establecimiento de especies alóctonas y no alteró la composición de la comunidad vegetal. Sin embargo, este resultado puede no ser extrapolable a áreas donde la vegetación se regenere por semilla. Se recomienda el empleo de mulch del sitio afectado y tener en cuenta su origen para evitar posibles efectos indeseados sobre la vegetación.

\section{Agradecimientos}

Este trabajo fue financiado por el proyecto INIA- RTA2014-00011-C06-02 e INDITEX.

\section{Referencias bibliográficas}

Bautista, S., Bellot, J., Vallejo, V.R., 1996. Mulching treatment for postfire soil conservation in a semiarid ecosystem. Arid Soil Research and Rehabilitation 10, 235-242.

Bautista, S., Robichaud, P.R., Bladé, C., 2009. Post-fire mulching. In: Cerdá, A., Robichaud, P.R. (Eds.), Fire effects on soils and restoration strategies. Science Publishers, Enfield, NH, USA, pp. 353-372.

Beyers, J., 2004. Postfire seeding for erosion control: Effectiveness and impacts on native plant communities. Conservation Biology 18, 947-956.

Core Team Development, R., 2014. R: A language and environment for statistical computing. In. R Foundation for Statistical Computing, Vienna.

De Bano, L.F., Neary, D.G., Ffolliott, P.F., 1998. Fire's effects on ecosystems. John Wiley and sons, New York.

Dodson, E.K., Peterson, D.W., 2010. Mulching effects on vegetation recovery following high severity wildfire in north-central Washington State, USA. Forest Ecology and Management. 260, 1816-1823.

Dodson, E.K., Peterson, D.W., Harrod, R.J., 2010. Impacts of erosion control treatments on native vegetation recovery after severe wildfire in the Eastern Cascades, USA. International Journal of Wildland Fire. 19, 490-499.

Fernández, C., Vega, J.A., 2014. Efficacy of bark strands and straw mulching after wildfire in NW Spain: Effects on erosion control and vegetation recovery. Ecological Engineering. 63, 50-57.

Fernández, C., Vega, J.A., Fonturbel, T., 2013. Does fire severity influence shrub resprouting after spring prescribed burning? Acta Oecologica. 48, 30-36.

Fernández, C., Vega, J.A., Jiménez, E., Fonturbel, T., 2011. Effectiveness of three post-fire treatments at reducing soil erosion in Galicia (NW Spain). International Journal of Wildland Fire. 20, 104-114.

Foltz, R.B., 2012. A comparison of three erosion control mulches on decommissioned forest road corridors in the northern Rocky Mountains, United States. Journal of Soil and Water Conservation. 67, 536-544.

Hunter, M.E., Omi, P.N., Martinson, E.J., Chong, G.W., 2006. Establishment of non-native plant species after wildfires: effects of fuel treatments, abiotic and biotic factors, and 
post-fire grass seeding treatments. International Journal of Wildland Fire. 15, 271-281. Johansen, M.P., Hakonson, T.E., Breshears, D.D., 2001. Post-fire runoff and erosion from rainfall simulation: contrasting forests with shrublands and grasslands. Hydrological Processes. 15, 2953-2965.

Kruse, R., Bend, E., Bierzychudek, P., 2004. Native plant regeneration and introduction of non-natives following post-fire rehabilitation with straw mulch and barley seeding. $\mathrm{FO}_{\mathrm{O}}$ rest Ecology and Management. 196, 299-310.

Napper, C., 2006. Burned Area Emergency Response treatments catalog. USDA Forest Service. National Technology \& Development Program. Watershed, Soil, Air Management 0625 1801-SDTDC.

Peterson, D.W., Dodson, E.K., Harrod, R.J., 2009. Fertilization and seeding effects on vegetative cover after wildfire in north-central Washington state. Forest Science. 55, 494502.

Neary, D.G., Ryan, K.C., De Bano, L.F., 2005. Wildland fire in ecosystems. Effects of fire on soil and water. Gen. Tech. Rep. RMRS-GTR-42

Reyes, O., Casal, M., 2008. Regeneration models and plant regenerative types related to the intensity of fire in Atlantic shrubland and woodland species. Journal of Vegetation Science. 19, 575-583.

Robichaud, P.R., Jordan, P., Lewis, S.A., Ashmun, L.E., Covert, S.A., Brown, R.E., 2013. Evaluating the effectiveness of wood shred and agricultural straw mulches as a treatment to reduce post-wildfire hillslope erosion in southern British Columbia, Canada. Geomorphology. 197, 21-33.

Santana, V.M., Alday, J.G., Baeza, M.J., 2014. Mulch application as post-fire rehabilitation treatment does not affect vegetation recovery in ecosystems dominated by obligate seeders. Ecological Engineering. 71, 80-86.

Sanz, M., Dana, E.D., Sobrino, E., 2004. Atlas de las Plantas Alóctonas Invasoras en Espa$\tilde{n} a$. Dirección General para la Biodiversidad, Madrid.

Shakesby, R.A., Doerr, S.H., 2006. Wildfire as a hydrological and geomorphological agent. Earth-Science Reviews. 74, 269-307.

Smets, T., Poesen, J., Knapen, A., 2008. Spatial scale effects on the effectiveness of organic mulches in reducing soil erosion by water. Earth-Science Reviews. 89, 1-12.

Vega, J.A., Fernández, C., Fontúrbel, M.T., González-Prieto, S.J., Jiménez, E., 2014. Testing the effects of straw mulching and herb seeding on soil erosion after fire in a gorse shrubland. Geoderma. 223-225, 79-87.

Vega, J.A., Fernández, C., Fonturbel, T., 2015. Comparing the effectiveness of seeding and mulching+seeding in reducing soil erosion after a high severity fire in Galicia (NW Spain). Ecological Engineering. 74, 206-212.

Vega, J.A., Fontúrbel, M.T., Fernández, C., Arellano, A., Díaz-Raviña, M., Carballas, T., Martín, A., González-Prieto, S., Merino, A., Benito, E., 2013. Acciones urgentes contra la erosión en áreas forestales quemadas: Guía para su planificación en Galicia. Santiago de Compostela. 